\title{
Mycobacterium algericum sp. nov., a novel rapidly growing species related to the Mycobacterium terrae complex and associated with goat lung lesions
}

Correspondence

Jakob Zinsstag

jakob.zinsstag@unibas.ch

\author{
Naima Sahraoui, ${ }^{1} \dagger$ Marie Ballif, ${ }^{2,3}+$ Samir Zelleg, ${ }^{1}$ Nadir Yousfi, ${ }^{1}$ \\ Claudia Ritter, ${ }^{4,5}$ Ute Friedel, ${ }^{4,5}$ Beat Amstutz, ${ }^{4,5}$ Djamel Yala, ${ }^{6}$ \\ Fadila Boulahbal, ${ }^{6}$ Djamel Guetarni, ${ }^{1}$ Jakob Zinsstag ${ }^{2,3}$ \\ and Peter M. Keller ${ }^{4,5}$ \\ ${ }^{1}$ Veterinary Department, University Saad Dahlab, Blida, Algeria \\ ${ }^{2}$ Swiss Tropical and Public Health Institute, Basel, Switzerland \\ ${ }^{3}$ University of Basel, Switzerland \\ ${ }^{4}$ Institute of Medical Microbiology, University of Zurich, Zurich, Switzerland \\ ${ }^{5}$ Swiss National Reference Centre for Mycobacteria, Zurich, Switzerland \\ ${ }^{6}$ Institut Pasteur, Algiers, Algeria
}

Non-tuberculous mycobacteria are typically found in the environment, but can be the cause of occasional opportunistic infections in humans or animals (Tortoli, 2009).

Members of the Mycobacterium terrae complex (Mycobacterium terrae sensu stricto, Mycobacterium nonchromogenicum, Mycobacterium triviale and Mycobacterium senuense) are ubiquitous and potentially opportunistic pathogens. Indeed, some reports showed potential human pathogenicity (Krisher et al., 1988; Mayo et al., 1998; Smith et al., 2000). Disseminated M. terrae infections have also been reported among AIDS patients (Carbonara et al., 2000). M. senuense was recently described as a novel Mycobacterium species closely related to the $M$. terrae

†These authors contributed equally to this work.

The GenBank/EMBL/DDBJ accession numbers for the 16S rRNA, $h s p 65$ and partial $r p o B$ gene sequences of strain TBE $500028 / 10^{\top}$ are GU564404, GU564405 and GU564406, respectively.

A supplementary table is available with the online version of this paper. complex (Mun et al., 2008). It was isolated from a Korean patient with a symptomatic pulmonary infection.

In this report, we present a previously undescribed Mycobacterium species, for which we propose the name Mycobacterium algericum sp. nov. (type strain, TBE $500028 / 10^{\mathrm{T}}$ ). Previous publications have shown that phenotypic and biochemical analyses often do not provide an accurate identification of Mycobacterium species (Cloud et al., 2006; Lee et al., 2004; Mun et al., 2008; Springer et al., 1996). Therefore, using a combination of both biochemical and molecular techniques, we showed that isolate TBE $500028 / 10^{\mathrm{T}}$, collected from a pulmonary lesion on a goat in Algeria, was representative of a novel Mycobacterium species related to the $M$. terrae complex, in particular M. terrae sensu stricto and M. senuense.

The isolate was collected in the Bejaia department (Wilaya) in Algeria in 2008, at the slaughterhouse of Souk El Tenine. Lung lesions observed on goat carcasses were sampled and sent to the Pasteur Institute in Alger. There, Ziehl-Neelsen 
staining and microscopy were performed. After grinding and decontamination by Petroff's method $(4 \% \mathrm{NaOH})$ (Ghosh et al., 1978; Petroff, 1915), samples were inoculated on Löwenstein-Jensen growth medium. Tubes were incubated at $37^{\circ} \mathrm{C}$ (ambient atmosphere) and monitored weekly until colonies suggesting mycobacterial growth were observed. Tubes with potential mycobacterial colonies were sent to Switzerland for further biochemical and molecular characterization of the isolate.

Biochemical characterization of strain TBE $500028 / 10^{\mathrm{T}}$ was performed by observing growth under various conditions, according to methods described by Murray et al. (2007); the determined characteristics were compared with those of the closely related Mycobacterium species M. terrae and $M$. senuense. To determine the optimal growth temperature, Middlebrook 7H10 agar plates were inoculated and incubated at temperatures ranging from $25{ }^{\circ} \mathrm{C}$ (with and without light) to $45{ }^{\circ} \mathrm{C}$ (ambient atmosphere). We also tested growth ability on MacConkey agar with and without $5 \% \mathrm{NaCl}$ at $37{ }^{\circ} \mathrm{C}$ (ambient atmosphere) and tellurite reduction, as well as nitrate reductase, urease, catalase and pyrazinamidase production. Colony morphology and pigmentation are described. Cell morphological analyses were conducted after growth in 7H9 liquid broth with $10 \%$ OADC supplement (oleic acid, bovine albumin, glucose and catalase). Cells were harvested in the exponentialgrowth phase and stained with auramine-rhodamine. Microscopic imaging was performed on a Zeiss Axiovert 200M microscope using a 100-fold magnifying objective (Carl Zeiss). Cell dimensions were determined by using the measurement tool provided by the SlideBook version 4.1 software (Intelligent Imaging Innovations). Acid-alcohol fastness was assessed by Ziehl-Neelsen staining.

Susceptibility to the antibiotics rifampicin (1.0 and $\left.10.0 \mathrm{mg} \mathrm{l}^{-1}\right)$, rifabutin $\left(0.1\right.$ and $\left.1.0 \mathrm{mg} \mathrm{l}^{-1}\right)$, ethambutol (5.0 and $50 \mathrm{mg} \mathrm{l}^{-1}$ ), clarithromycin $(4.0,16.0,32.0$ and $\left.64.0 \mathrm{mg} \mathrm{l}^{-1}\right)$, amikacin $\left(1.0\right.$ and $\left.10 \mathrm{mg} \mathrm{l}^{-1}\right)$, ofloxacin $(2.0$ and $\left.10.0 \mathrm{mg} \mathrm{l}^{-1}\right)$ and moxifloxacin $\left(0.5,2.5\right.$ and $\left.10 \mathrm{mg} \mathrm{l}^{-1}\right)$ was tested in mycobacterial growth indicator tubes (MGIT 960; Becton Dickinson) and monitored with EpiCenter software and the TB eXiST module (Springer et al., 2009).

HPLC analyses were performed to investigate the profile of cell-wall mycolic acids (Butler \& Guthertz, 2001; CDC, 1996). The profile obtained for our isolate was compared with those from other closely related Mycobacterium species.

DNA was extracted from cultures using the Bio-Rad InstaGene Matrix. In order to assess the phylogenetic relationships of the isolate, we performed sequencing of the nearly complete 16S rRNA gene $(1521 \mathrm{bp}$, corresponding to Mycobacterium tuberculosis $\mathrm{H}_{37 \mathrm{Rv}^{\mathrm{T}}}$ rrs nt 18 1534) using previously described primers and protocols (Rogall et al., 1990; Springer et al., 1996) and two new primers (16s_742_fw, 5' ${ }^{\prime}$-AGCGTGGGGAGCGAACAGG-3'; 16s_1408_rv, 5'-CCCGAAGCCGGTGGCCTAA-3'). Two regions of the $r p o B$ gene were amplified and sequenced:
710 bp (M. tuberculosis $\mathrm{H}_{37 \mathrm{Rv}^{\mathrm{T}}}$ rpoB nt 2436-3145) according to Adékambi et al. (2003), and 553 bp (M. tuberculosis $\mathrm{H}_{37 \mathrm{Rv}^{\mathrm{T}}} r p o B$ nt 1092-1645) according to Kim et al. (1999), with a modified reverse primer: MR2, 5'-CAGCTCGTCGTCGTCCTC-3'. A 441 bp part of the hsp65 gene (M. tuberculosis $\mathrm{H}_{37 \mathrm{Rv}^{\mathrm{T}}}$ groEL2 nt 145-585) was sequenced according to Telenti et al. (1993). All amplifications included the reference strain $M$. tuberculosis $\mathrm{H} 37 \mathrm{Rv}^{\mathrm{T}}$ as positive control, and buffers without any added mycobacterial DNA as negative control.

Nucleotide sequences were obtained for both forward and reverse primers. Sequence contingency alignment was performed for each gene with the software SeqMan version 7.0 (DNASTAR Inc.). The obtained consensus sequences were compared with mycobacterial sequences by similarity search in GenBank, using the BLASTN algorithm. Phylogenetic trees comparing our isolate with other Mycobacterium species were obtained for each gene by using the MEGALIGN software (DNASTAR Inc.) with default settings for CLUSTAL V neighbour-joining multiple alignments. Note that the $r p o B$ phylogeny was calculated based on the 553 bp sequence fragment. All obtained trees were established by bootstrap analyses with 1000 resamplings and 111 seeds.

The goat from which the isolation was made presented nodular lung lesions of caseous aspect. Direct microscopy of Ziehl-Neelsen-stained tissues showed low-density (six acid-alcohol-resistant bacilli in 300 observed fields) infections with slightly curved bacilli. The isolate was inoculated into Löwenstein-Jensen tubes in Algeria. Growth rate was slow on Löwenstein-Jensen medium: small, round, white-yellow colonies were observed after 35 days. Inversely, subculture on Middlebrook 7H10 agar plates was rapid; microcolonies could be observed after 1 week at temperatures ranging between 25 and $40{ }^{\circ} \mathrm{C}$, with or without light. Optimal growth was seen at 37 and $40{ }^{\circ} \mathrm{C}$, whereas no growth was observed at $45{ }^{\circ} \mathrm{C}$. On Middlebrook $7 \mathrm{H} 10$ agar plates, colonies were small, polymorphic, white, non-chromogenic, smooth and with irregular edges. Ziehl-Neelsen and auramine-rhodamine staining showed acid-fast, $1.4 \pm 0.2 \mu \mathrm{m}$ long, rod-shaped bacilli. No spores, cords or filaments were observed.

No growth was observed on MacConkey agar with or without $5 \% \mathrm{NaCl}$. Production of urease, a positive nitrate reductase reaction, a positive catalase reaction at $25^{\circ} \mathrm{C}$, and tellurite reductase activity were observed.

Drug-susceptibility testing in MGIT 960 showed susceptibility to all drugs tested (see Supplementary Table S1, available in IJSEM Online), with the exception of low-level resistance to aminoglycosides ( $1.0 \mathrm{mg}$ amikacin $\mathrm{l}^{-1}$ ), quinolones $\left(0.5 \mathrm{mg}\right.$ moxifloxacin $\mathrm{l}^{-1}$ and $2.0 \mathrm{mg}$ ofloxacin $\left.\mathrm{l}^{-1}\right)$ and rifampicin $\left(1.0 \mathrm{mg} \mathrm{l}^{-1}\right)$. Pyrazinamidase production was detected. Strain TBE $500028 / 10^{\mathrm{T}}$ is thus sensitive to pyrazinamide.

HPLC analysis of the cell wall's mycolic acids demonstrated a unique profile. Although related closely to the profiles 


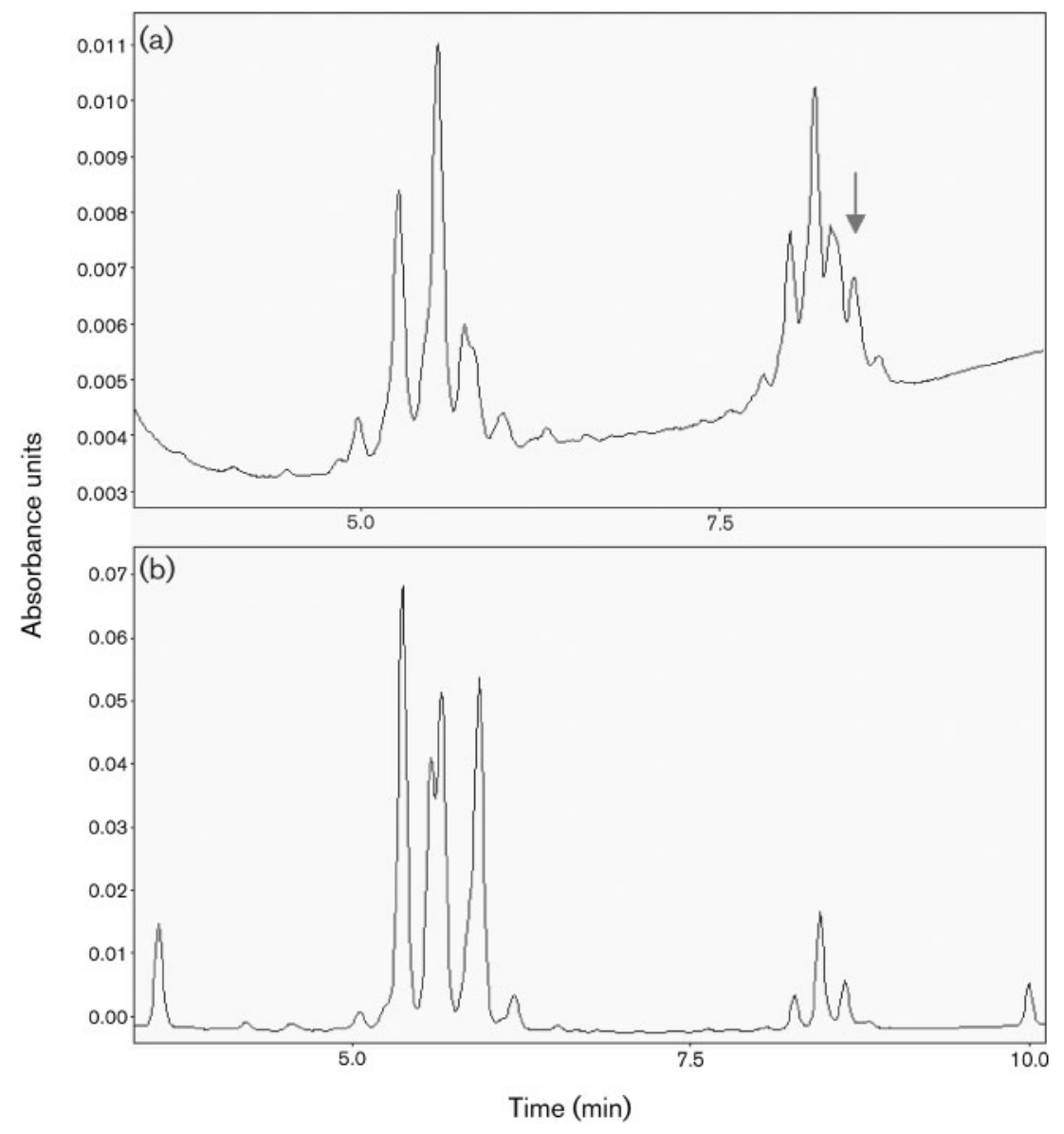

Fig. 1. Mycolic acid profiles obtained by HPLC. (a) TBE 500028/10 ${ }^{\top}$; (b) M. terrae ATCC $15755^{\top}$. The arrow in (a) indicates an additional peak in the late cluster of the profile of strain TBE $500028 / 10^{\top}$. observed for other $M$. terrae complex subspecies, the chromatogram obtained for our isolate distinctively presented an additional peak in the late cluster (Fig. 1).

The overall biochemical profile of the isolate was similar to those of $M$. terrae and $M$. senuense, with the exception of an intermediate instead of a slow growth rate at $37{ }^{\circ} \mathrm{C}$ (Table 1). Furthermore, in contrast to the type strains of $M$. terrae and M. senuense, strain TBE $500028 / 10^{\mathrm{T}}$ had a positive urease activity and a different antibiotic-susceptibility pattern (Mun et al., 2008; Murray et al., 2007; Smith et al., 2000). Indeed, unlike strain TBE 500028/10 ${ }^{\mathrm{T}}$, M. terrae and M. senuense are susceptible to rifampicin, amikacin and quinolones (M. senuense only).

Based on the BLAST sequence match obtained for the $16 \mathrm{~S}$ rRNA gene (GenBank accession no. GU564404), strain TBE $500028 / 10^{\mathrm{T}}$ was shown to be most closely related to M. senuense $05-832^{\mathrm{T}}(1492 / 1522 \mathrm{nt}, 98.0 \%)$, M. terrae ATCC $15755^{\mathrm{T}}(1451 / 1470 \mathrm{nt}, 98.7 \%)$ and Mycobacterium arupense AR30097 ${ }^{\mathrm{T}}(1458 / 1495 \mathrm{nt}, 97.5 \%)$. The two combined partial sequences of the $r p o B$ gene (GenBank accession no. GU564406) showed that strain TBE 500028/ $10^{\mathrm{T}}$ was related most closely to $M$. senuense $05-832^{\mathrm{T}}(403 /$ $421 \mathrm{nt}, 95.7 \%)$, M. terrae ATCC $15755^{\mathrm{T}}(591 / 633 \mathrm{nt}$, 93.4\%), Mycobacterium confluentis CIP $105510^{\mathrm{T}}(632 /$ $703 \mathrm{nt}, 89.9 \%)$ and Mycobacterium chitae CIP $105383^{\mathrm{T}}$
(632/703 nt, 89.9\%). The hsp65 gene sequence (GenBank accession no. GU564405) showed that our strain was related most closely to $M$. senuense strain DSM $44999^{\mathrm{T}}$ (421/431 nt, $97.7 \%$ ) and M. terrae strain CIP $104321^{\mathrm{T}}$ (414/426 nt, $97.2 \%)$.

Table 1. Cultural and biochemical characteristics for $M$. algericum sp. nov. in comparison with the closely related $M$. terrae and $M$. senuense

Taxa: 1 , M. algericum TBE 500028/10 ${ }^{\mathrm{T}} ; 2$, M. terrae ATCC $15755^{\mathrm{T}}$; 3, M. senuense DSM $44999^{\mathrm{T}}$. Data for $M$. terrae and M. senuense were retrieved from the literature (Mun et al., 2008; Murray et al., 2007). + , Positive; -, negative; + /-, variable; I, intermediate in roughness; $\mathrm{S}$, smooth; W, white; Y, yellow. All taxa were non-photochromogenic, negative for growth at $45{ }^{\circ} \mathrm{C}$ and on MacConkey agar both with and without $5 \% \mathrm{NaCl}$, and positive for production of nitrate reductase, catalase, tellurite reductase and pyrazinamidase.

\begin{tabular}{|lccc|}
\hline Characteristic & $\mathbf{1}$ & $\mathbf{2}$ & $\mathbf{3}$ \\
\hline Growth within 7 days at $37{ }^{\circ} \mathrm{C}$ & + & $+/-$ & - \\
Optimal growth temperature $\left({ }^{\circ} \mathrm{C}\right)$ & $37-40$ & 35 & 37 \\
Colony morphology on $7 \mathrm{H} 10$ & IW & IWY & SWY \\
Urease production & + & - & - \\
\hline
\end{tabular}


The close relationship to $M$. terrae and $M$. senuense was confirmed by phylogenetic analyses (Fig. 2). Trees based on $r p o B$ and $h s p 65$ gene sequences each showed similar groupings for strain TBE $500028 / 10^{\mathrm{T}}$, but slightly different from that of the $16 \mathrm{~S}$ rRNA gene tree. In the $r p o B$ and $h s p 65$ trees, TBE $500028 / 10^{\mathrm{T}}$ forms a branch dependent of M. terrae together with $M$. senuense, whereas in the $16 \mathrm{~S}$ rRNA gene tree, strain $500028 / 10^{\mathrm{T}}$ forms a branch directly
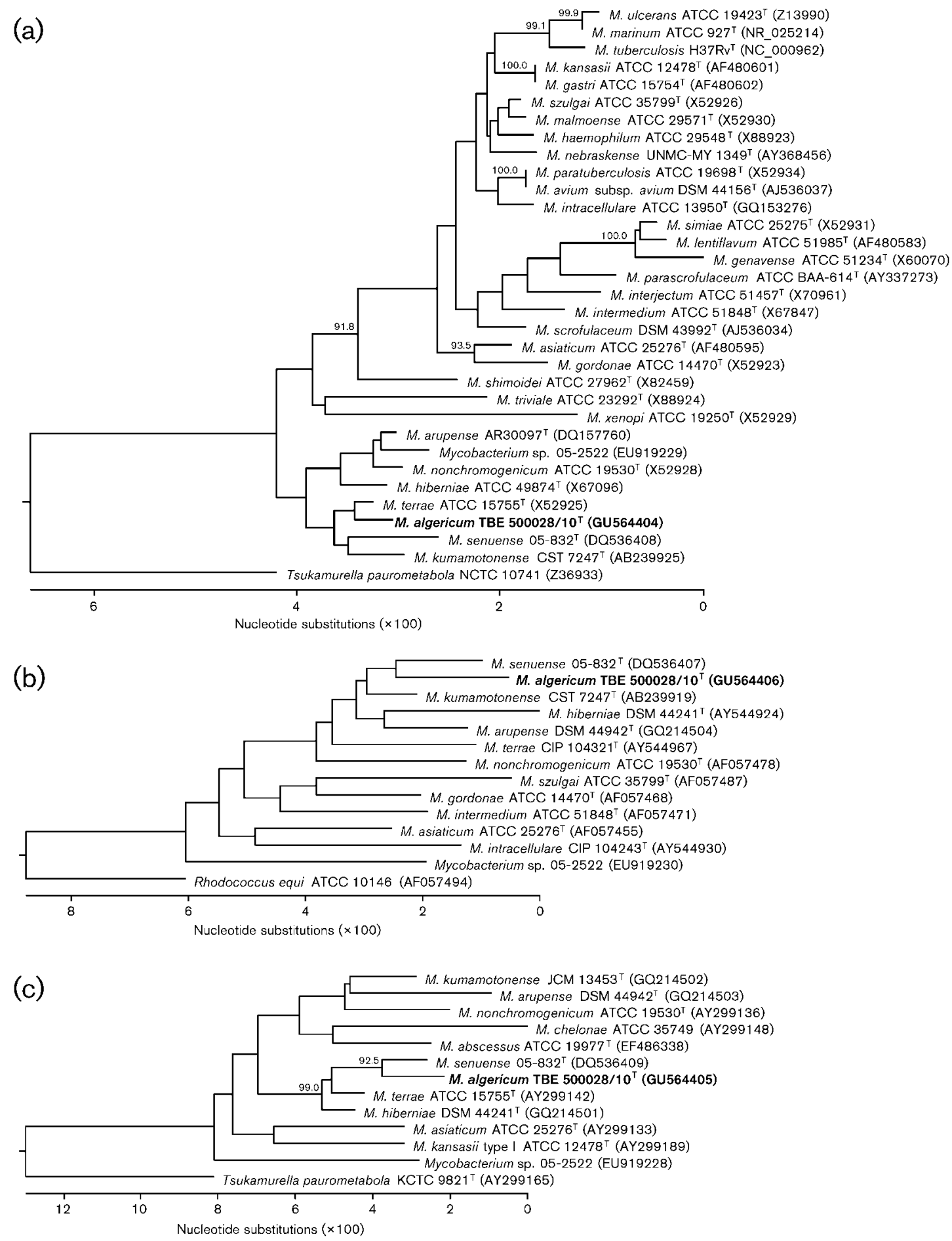

Fig. 2. Phylogenetic trees designed with the neighbour-joining method and bootstrapped 1000 times. Bootstrapping values $>90 \%$ are indicated at nodes. Trees were based on: (a) $16 \mathrm{~S}$ rRNA gene; (b) 553 bp partial sequence of the rpo $B$ gene; (c) hsp65 gene. 
dependent from M. terrae, with M. senuense being situated in another cluster. These topologies were also supported by high bootstrap values.

The species which our isolate resembles most closely, $M$. terrae and $M$. senuense, are both slow-growing, non-chromogenic and potentially pathogenic to humans.

\section{Description of Mycobacterium algericum sp. nov.}

Mycobacterium algericum (al.ge'ri.cum. N.L. neut. adj. algericum pertaining to Algeria, the country where the strain was first isolated).

Bacillus that stains acid-alcohol-fast. Cells are rod-shaped and $1.4 \pm 0.2 \mu \mathrm{m}$ long. Cording, spores and filaments are not observed. On Middlebrook 7H10 agar plates, growth is fast, with optimal growth between 37 and $40{ }^{\circ} \mathrm{C}$. Microcolonies can be observed after 1 week. However, 5 weeks are necessary to observe colonies on LöwensteinJensen medium. Colonies on Middlebrook 7H10 agar plates are small, polymorphic, white, non-chromogenic, smooth and with irregular edges. Colonies grown on LöwensteinJensen medium are small, round and white-yellow. No growth is observed on MacConkey agar plates with or wthout $5 \% \mathrm{NaCl}$. Urease, nitrate reductase, catalase and tellurite reductase production is positive. Pyrazinamidase production is positive. Susceptible to clarithromycin, ethambutol and rifabutin, but low-level resistant to amikacin, moxifloxacin, ofloxacin and rifampicin. HPLC analysis of mycolic acids showed a unique profile. Genetically, 16S rRNA, rpoB and $h s p 65$ gene sequences are unique. Phylogenetic analyses showed that the species is related to $M$. terrae sensu stricto and $M$. senuense.

The type strain is TBE $500028 / 10^{\mathrm{T}} \quad\left(=\right.$ Bejaia $^{\mathrm{T}}=\mathrm{CIP}$ $110121^{\mathrm{T}}=$ DSM $45454^{\mathrm{T}}$ ), isolated from a lung lesion of an Algerian goat.

\section{Acknowledgements}

The University of Zurich supported this study technically and financially. M. B. was funded by the Swiss National Science Foundation (grant no. IZ 70-Z0-123988). For the HPLC profile, we thank Professor Enrico Tortoli, Mycobacteria Reference Center, Florence, Italy.

\section{References}

Adékambi, T., Colson, P. \& Drancourt, M. (2003). rpoB-based identification of nonpigmented and late-pigmenting rapidly growing mycobacteria. J Clin Microbiol 41, 5699-5708.

Butler, W. R. \& Guthertz, L. S. (2001). Mycolic acid analysis by high-performance liquid chromatography for identification of Mycobacterium species. Clin Microbiol Rev 14, 704-726.

Carbonara, S., Tortoli, E., Costa, D., Monno, L., Fiorentino, G., Grimaldi, A., Boscia, D., Rollo, M. A., Pastore, G. \& Angarano, G. (2000). Disseminated Mycobacterium terrae infection in a patient with advanced human immunodeficiency virus disease. Clin Infect Dis 30, 831-835.

CDC (1996). Standardized Method for HPLC Identification of Mycobacteria. Atlanta, GA: US Department of Health and Human Services, Public Health Service.

Cloud, J. L., Meyer, J. J., Pounder, J. I., Jost, K. C., Jr, Sweeney, A., Carroll, K. C. \& Woods, G. L. (2006). Mycobacterium arupense sp. nov., a non-chromogenic bacterium isolated from clinical specimens. Int $J$ Syst Evol Microbiol 56, 1413-1418.

Ghosh, H. K., Cobb, M., Pacey, D. P. \& Conklin, S. (1978). Experience with a simplification of the Petroff method for laboratory diagnosis of mycobacteria in sputum. Pathology 10, 257-261.

Kim, B. J., Lee, S. H., Lyu, M. A., Kim, S. J., Bai, G. H., Chae, G. T., Kim, E. C., Cha, C. Y. \& Kook, Y. H. (1999). Identification of mycobacterial species by comparative sequence analysis of the RNA polymerase gene (rpoB). J Clin Microbiol 37, 1714-1720.

Krisher, K. K., Kallay, M. C. \& Nolte, F. S. (1988). Primary pulmonary infection caused by Mycobacterium terrae complex. Diagn Microbiol Infect Dis 11, 171-175.

Lee, C. K., Gi, H. M., Cho, Y., Kim, Y. K., Lee, K. N., Song, K. J., Song, J. W., Park, K. S., Park, E. M. \& other authors (2004). The genomic heterogeneity among Mycobacterium terrae complex displayed by sequencing of $16 \mathrm{~S}$ rRNA and hsp 65 genes. Microbiol Immunol 48, 83-90.

Mayo, J., Collazos, J. \& Martínez, E. (1998). Mycobacterium nonchromogenicum bacteremia in an AIDS patient. Emerg Infect Dis 4, 124-125.

Mun, H. S., Park, J. H., Kim, H., Yu, H. K., Park, Y. G., Cha, C. Y., Kook, Y. H. \& Kim, B. J. (2008). Mycobacterium senuense sp. nov., a slowly growing, non-chromogenic species closely related to the Mycobacterium terrae complex. Int J Syst Evol Microbiol 58, 641-646.

Murray, P. R., Baron, E. J., Jorgensen, J. H., Landry, M. L. \& Pfaller, M. A. (2007). Manual of Clinical Microbiology, 9th edn, pp. 543-588. Washington, DC: American Society for Microbiology.

Petroff, S. A. (1915). A new and rapid method for the isolation and cultivation of tubercle bacilli directly from the sputum and feces. J Exp Med 21, 38-42.

Rogall, T., Flohr, T. \& Böttger, E. C. (1990). Differentiation of Mycobacterium species by direct sequencing of amplified DNA. J Gen Microbiol 136, 1915-1920.

Smith, D. S., Lindholm-Levy, P., Huitt, G. A., Heifets, L. B. \& Cook, J. L. (2000). Mycobacterium terrae: case reports, literature review, and in vitro antibiotic susceptibility testing. Clin Infect Dis 30, 444453.

Springer, B., Stockman, L., Teschner, K., Roberts, G. D. \& Böttger, E. C. (1996). Two-laboratory collaborative study on identification of mycobacteria: molecular versus phenotypic methods. J Clin Microbiol 34, 296-303.

Springer, B., Lucke, K., Calligaris-Maibach, R., Ritter, C. \& Böttger, E. C. (2009). Quantitative drug susceptibility testing of Mycobacterium tuberculosis by use of MGIT 960 and EpiCenter instrumentation. J Clin Microbiol 47, 1773-1780.

Telenti, A., Marchesi, F., Balz, M., Bally, F., Böttger, E. C. \& Bodmer, T. (1993). Rapid identification of mycobacteria to the species level by polymerase chain reaction and restriction enzyme analysis. J Clin Microbiol 31, 175-178.

Tortoli, E. (2009). Clinical manifestations of nontuberculous mycobacteria infections. Clin Microbiol Infect 15, 906-910. 